\title{
Kundt geometries and memory effects in the Brans-Dicke theory of gravity
}

\author{
Siddhant Siddhant ${ }^{\mathrm{a}}$, Indranil Chakraborty ${ }^{\mathrm{b}}$, Sayan Kar ${ }^{\mathrm{c}}$ \\ Department of Physics, Indian Institute of Technology Kharagpur, Kharagpur 721 302, India
}

Received: 3 December 2020 / Accepted: 6 April 2021 / Published online: 23 April 2021

(C) The Author(s) 2021

\begin{abstract}
Memory effects are studied in the simplest scalar-tensor theory, the Brans-Dicke (BD) theory. To this end, we introduce, in BD theory, novel Kundt spacetimes (without and with gyratonic terms), which serve as backgrounds for the ensuing analysis on memory. The BD parameter $\omega$ and the scalar field $(\phi)$ profile, expectedly, distinguishes between different solutions. Choosing specific localised forms for the free metric functions $H^{\prime}(u)$ (related to the wave profile) and $J(u)$ (the gyraton) we obtain displacement memory effects using both geodesics and geodesic deviation. An interesting and easy-to-understand exactly solvable case arises when $\omega=-2$ (with $J(u)$ absent) which we discuss in detail. For other $\omega$ (in the presence of $J$ or without), numerically obtained geodesics lead to results on displacement memory which appear to match qualitatively with those found from a deviation analysis. Thus, the issue of how memory effects in BD theory may arise and also differ from their GR counterparts, is now partially addressed, at least theoretically, within the context of this new class of Kundt geometries.
\end{abstract}

\section{Introduction}

The detection of gravitational waves in binary mergers has opened up new prospects for testing theories of gravity in the strong field regime [1,2]. Gravitational wave memory is one such as-yet-unobserved strong field effect that can be used to test diverse theories of gravity. The gravitational wave memory effect is the residual permanent shift in the position (or velocity) caused due to the passage of a gravitational wave pulse [3].

The study of memory effects began in the work of Zel'dovich and Polnarev [4] who studied gravitational radia-

\footnotetext{
a e-mail: siddhant@iitkgp.ac.in

b e-mail: indradeb@iitkgp.ac.in (corresponding author)

c e-mail: sayan@phy.iitkgp.ac.in
}

tion emitted due to the motion of flybys/collapse of stars in a globular cluster. A few years later, Braginsky and Grishchuk [5] looked at the deviation of test particles in weak field, linearized gravity and coined the term memory effect to denote the change in the metric perturbation at early and late times. Geodesic deviation of test particles due to low frequency gravitational radiation at null infinity was investigated further by Ludvigsen [6]. Christodoulou, using full nonlinear GR, attributed the presence of memory to the transport of energy and momentum of gravitational waves to null infinity [7]. Further, this effect, related to non-linearity, was ascribed to gravitons produced by the radiation itself [8]. Memory effects are also possible in electrodynamics [9] and YangMills theories [10,11]. Interesting theoretical links to memory effects have been conjectured, of late, in the context of soft theorems and BMS symmetries [12]. It has been noted that the nonlinear memory effect can also be understood as a BMS transformation relating two inequivalent Minkowski vacua at future null infinity caused by the passage of gravitational waves (see the review [13] and the references cited therein).

Apart from spacetime boundaries, memory effects can also be realized in the interior regions of a spacetime $[14,15]$. Such effects lead to permanent changes due to presence of gravitational waves and have been termed as persistent observables in [16]. Our work mainly focuses on one such observable named displacement memory observable and tries to calculate the memory in non-flat Kundt geometries.

Memory effects in non-flat backgrounds in GR have been studied in both dS [17,18] (motivations from cosmology) and AdS spacetimes [19]. In [19], the authors have showed how to isolate the gravitational wave contribution from the background spacetime by resorting to Fermi normal coordinates and solving the geodesic deviation equation. They treated the wave as a perturbation over AdS spacetime caused due to scattering of massive/massless particles. In our work, we adopt the same method for studying memory effects in 
Kundt spacetimes. However, in our case, the setting is nonperturbative, since we deal with exact spacetimes representing gravitational waves.

Kundt spacetimes are exact radiative geometries consisting of non-expanding, non-shearing and non-twisting null geodesic congruences (NGC) [20-22]. They admit various wave solutions ( $p p$ waves, Siklos waves $[23,24]$ ) related to the presence of NGC whose tangent vector is usually not covariantly constant. In general, the wave surfaces may not be Cartesian planes. This non-planarity can signal the presence of matter or a cosmological constant [25]. Gyratons (spinning relativistic sources) are solutions obtained as a sub-class of Kundt geometries [26-28]. Presence of gyratonic matter in a Kundt geometry imparts an angular momentum due to its intrinsic spin. Till date, most of the research around Kundt geometries have largely been focused within the realm of GR [29-34], though there is some recent work in Gauss-Bonnet [35] and quadratic gravity [36]. As far as we know, not much has been done on such geometries within the ambit of scalar tensor theories. Our article is one such attempt towards (a) contructing new Kundt-type solutions in the simplest scalartensor theory, BD gravity and, more importantly, (b) verifying/contrasting the presence/absence of memory effects w.r.t similar ones in GR.

There does exist previous work on memory effects in BD theory which are different from what we wish to pursue here. As is well known, in linearised gravity, the BD scalar field produces a breathing mode along with the two additional polarizations $(+, \times)$ found in GR [37]. Lang computed GW waveforms for scalar and tensor modes separately in the PN approximation $[38,39]$. Du and Nishizawa proposed a test of gravity for scalar tensor theories [40]. They found two distinct sets of memory contributions: T-memory (tensor) and S-memory (scalar). Scalar memory is unique in such theories and was used as a tool to understand the Vainshtein screening mechanism in BD gravity [41]. Asymptotically flat spacetimes in BD theory and memory have been recently studied in $[42,43]$. The BMS group [44] is retained for the tensorial case. There are degenerate vacua for the scalar sector related via Lorentz transformations. The BMS charge algebra is computed in [45].

Studying memory effects for such Kundt wave spacetimes in GR was initiated by two of us in [46] through analysis of geodesic motion. Similar to exact plane wave spacetimes, one can construct sandwich waves here by choosing appropriate limiting profiles $[14,15,22]$. This serves as a qualitative toy model of a gravitational wave burst. Interesting distinctions occur between negative and positive constant curvature solutions, particularly for the latter, where we found a new frequency memory effect. In our analysis here, we consider memory in Kundt geometries without and with gyratonic terms. First, we construct explicit Kundt solutions for arbitrary $\omega$. The case $\omega=-2$ is special because it yields a spacetime with constant negative scalar curvature. For other $\omega$ we have variable positive or negative curvature. After constructing the solutions, we analyse geodesics with the intent of studying displacement memory. Thereafter, we move on to geodesic deviation. We do this by isolating the background, wave and gyratonic contributions to the deviation vector and their evolution equations. The coupled system of equations are then solved to obtain the behaviour of the deviation which helps us in analysing the presence of memory. We will also see how displacement memory is related to the BD scalar field and memory obtained via deviation shows the contributions of the background, gravitational wave and/or the gyratonic parts in the total deviation and hence, memory.

In our approach towards solving the deviation equation, our calculations are done in Fermi normal coordinates [19]. Here the coordinate system is locally Minkowskian and hence the notion of displacement and velocity memory effect is qualitatively similar to exact plane wave spacetimes $[15,47,48]$. In such Fermi coordinates, we construct parallely transported tetrads along a given timelike geodesic. The deviation vector is obtained w.r.t. the tetrad directions and then transformed back to the coordinate basis. We demonstrate the calculations in several specific cases (including an exactly solvable example) in the relevant section below.

The organization of our paper is as follows. In Sect. 2 we lay out the basic framework and the tools used in the paper. Section 3 deals with Kundt wave solutions without gyratonic terms and memory effects. Section 4 covers the Kundt metric with gyratonic terms and aspects of memory. We summarise our work in Sect. 5 with comments on possible extensions. Relevant mathematical formulae used in the paper are listed in an Appendix.

\section{Basic framework}

\subsection{Brans-Dicke gravity}

Brans and Dicke, seeking motivation from Mach's principle, proposed their alternative theory [49], where the Newtonian gravitational constant $(\mathrm{G})$ is related to the reciprocal of a scalar field. This link is based on the idea of variability of inertial mass at different points in spacetime. The action for the BD theory in the Jordan frame is given below.

$S=\int \sqrt{-g}\left[\phi R-\frac{\omega}{\phi} \nabla_{\alpha} \phi \nabla^{\alpha} \phi+16 \pi \mathcal{L}_{m}\right] d^{4} x$

Here, $\phi$ denotes the ambient scalar field, $\omega$ is the BD parameter and the $\mathcal{L}_{m}$ denotes the matter Lagrangian. $\omega$ is a dimensionless parameter which is highly constrained from Solar System observations [50]. Different values of $\omega$ correspond to different theories. 
Since its arrival on the scene in the early sixties, BD theory has been ruled out at times but has reappeared in different avatars serving diverse needs. For example, its $\omega=-1$ limit is dilaton gravity, which emerges from string theory [51]. Similarly, extensions such as replacing a constant $\omega$ with $\omega(\phi)$ leads to a broader class of theories which are actively pursued today in cosmological and astrophysical contexts [52]. Much of the relevance of BD, as well as scalar-tensor theories, rests on providing templates for comparative studies with GR, with the hope of discovering the new physics embodied in the theoretical constructs or ruling them out. Our work here, is also an attempt in a similar direction vis-a-vis the memory effect.

As mentioned before, we study Kundt geometries [20-22] in BD theory. Given our primary motivation with regard to distinguishing memory effects arising in GR and BD theory we first construct the solutions (Kundt-type). In our theoretical setup, we do not restrict the value of $\omega$. Instead we solve the field equations for novel Kundt geometries in BD theory and choose the value of $\omega$ to study specific cases. Interestingly, we find that in both the Kundt waves and gyratonic Kundt metric, the value of $\omega$ can be chosen freely. Hence, we analyse memory for specific values $\omega=-2,+1$. The reasons for choosing such specific values are explicitly discussed in Sect. 2.2.3. In all the cases considered here, we find vacuum solutions $\left(\mathcal{L}_{m}=0\right)$.

The field equations are obtained by variation of $g_{\mu \nu}$ and $\phi$. After performing a little algebra, we can write them in the standard form as shown below.

$G_{\mu \nu}=\frac{\omega}{\phi^{2}}\left[\phi,{ }_{\mu} \phi,{ }_{\nu}-\frac{1}{2} g_{\mu \nu} \phi,{ }_{\alpha} \phi,{ }^{\alpha}\right]+\frac{1}{\phi}\left(\phi, \mu ; \nu-g_{\mu \nu} \square \phi\right)$

$\square \phi=0$

The box operator is constructed using the Kundt spacetime metric.

\subsection{Kundt geometries and the geodesic analysis of memory}

Let us first introduce the class known as as Kundt geometries. We also point out how geodesic analysis leads to our understanding of memory effects for such geometries. Later in Sects. 3 and 4 we provide explicit calculations of memory for various solutions in BD theory which are special cases of the spacetimes mentioned below.

\subsubsection{Kundt wave metric}

The line element for a Kundt wave geometry is given as,

$$
d s^{2}=-H(u, x, y) d u^{2}-2 d u d v+\frac{d x^{2}+d y^{2}}{P(u, x, y)^{2}}
$$

The waves (denoted via the term $H(u, x, y)$ ) are viewed as propagating in the curved background spacetime $[25,30,46]$. The background curvature is dependent on $P(u, x, y)$.

\subsubsection{Kundt metric with gyraton/gyraton-like terms}

The line element for a generalisation of the metric above is given as,

$$
\begin{aligned}
d s^{2}= & -H d u^{2}-2 d u d v-2 W_{1} d u d x-2 W_{2} d u d y \\
& +\frac{1}{P^{2}}\left(d x^{2}+d y^{2}\right)
\end{aligned}
$$

$P \equiv P(u, x, y), H \equiv H(u, v, x, y), W_{i} \equiv W_{i}(u, v, x, y)$, $\forall i \in\{x, y\}$

The vector field $\mathbf{k}=\partial_{v}$ gives the NGC. The tangent to the spatial surfaces $\left(P \partial_{x}, P \partial_{y}\right)$ and $\mathbf{k}$ are orthogonal to each other. In this paper, we specifically work with gyratonic spacetimes where $W_{1}, W_{2}, H$ are independent of coordinate $v$ [26,28]. The off-diagonal $W_{i}$ act as sources of angular momentum in the spacetime and hence, such line elements correspond to spinning null sources. Note that Eq. (5) reduces to Eq. (4) if the cross terms $\left(W_{1}, W_{2}\right)$ are set to zero and $H,{ }_{v}=0$.

We obtain geometries in BD theory representing Kundt waves without and with gyratons beginning with metric ansatze given by Eqs. (4) or (5) respectively. The functional dependencies of $H$ and $W_{i}$ are chosen as: $H \equiv H(u, x, y)$ and $W_{i} \equiv W_{i}(u, x, y)$. In both cases, the BD parameter $(\omega)$ can be set by hand and is not constrained by the field equations. Using this freedom we can construct spacetimes with positive or negative curvature (Ricci scalar). Thereafter, we study geodesics and geodesic deviation to infer about memory effects.

\subsubsection{Geodesic analysis of memory}

Zhang et al. [14,47] have recently studied memory effects by analysing the evolution of geodesics in exact plane wave spacetimes. By choosing a Gaussian pulse for the polarization (radiative) term in the line element, they solved the geodesic equations numerically. The change in separation and velocity caused due to the passage of such a pulse was termed displacement and velocity memory effect. We further extended their analysis on predicting memory from geodesic analysis, for Kundt wave spacetimes in GR [46]. Expectedly, it was found that there exists a link between memory effects and the wave profile/curvature of the background spacetime. In order to test this hypothesis in an alternative theory of gravity, we construct and investigate memory for solutions in BD gravity with constant (negative) and varying (positive) curvature scalar. This indicates a direct comparison with results on memory in GR within the Kundt class of geometries. 


\subsection{Memory effects and geodesic deviation equation}

Apart from a geodesic analysis, one can understand memory from geodesic deviation. In one of the seminal works on memory effect, Braginsky and Grishchuk [5] studied geodesic deviation between two test particles moving in a weak gravitational wave. The set of equations following from geodesic deviation was recast into an equation of a forced system. The forcing was shown to be caused due to the passage of the gravitational wave. Integrating the forced equation one could obtain the change in separation between the two particles. This separation was caused only due to the gravitational wave and hence was a residual change (memory) imprinted on the spacetime.

In general, the deviation between two neighbouring geodesics in curved spacetimes gets contributions from both gravitational wave part and the non-flat background. Thus, recovering the deviation solely due to the wave (to find the memory effect) is nontrivial. A step along this direction of calculating memory effect in AdS spacetime was initiated in [19]. They considered linearized gravitational wave perturbations over AdS background spacetime. Constructing a parallelly propagated tetrad along a timelike geodesic, they employed Fermi normal coordinates to separate the gravitational wave part from the background. We use the same method as given in [19] for our analysis. The only difference here is that in our case the spacetime itself is a radiative geometry while in the former case the perturbations act over a nonradiative background (i.e. AdS). Fermi coordinates were also used in [48] to find the memory for gravitational shock waves and gyratonic pp-waves. Physically it corresponds to a set of timelike inertial observers traversing along the central geodesic whose spatial distance scales are very small compared to the curvature length scale.

After obtaining the geodesic deviation in the tetrad basis, we transform them back to the coordinate basis. Thus, we determine separately, deviation arising due to different sources (e.g. background or the wave) in the coordinate basis. The total deviation will, of course, be qualitatively similar to results obtained from the geodesic analysis.

Let us now explicitly define Fermi normal coordinates and parallelly transported tetrads, which we will use to arrive at memory effects. Consider the geodesic deviation equation

$\frac{D^{2}}{d \lambda^{2}} \xi^{\mu}=-R^{\mu}{ }_{\nu \rho \sigma} U^{\nu} \xi^{\rho} U^{\sigma}$

Here, $\xi^{\mu}$ is the deviation vector between neighbouring timelike geodesics. $U^{\mu}$ is the tangent vector along one of the geodesics. $R^{\mu}{ }_{\nu \rho \sigma}$ being the Riemann curvature tensor. Along a chosen geodesic one can set up a coordinate system $\left\{t, Z^{i}\right\}$ such that the Christoffel connections are always zero along that curve. The spacetime curvature manifests itself through the Riemann curvature. Such basis sets are known as Fermi bases [53]. Along the geodesic, $t$ denotes the proper time and $Z^{i}=0$. A parallely transported tetrad is denoted as $e^{\mu} a$. This satisfies

$U^{\alpha} \nabla_{\alpha} e^{\mu}=0$

Construction of such a tetrad depends on the spacetime metric. In the tetrad $e^{\mu}{ }_{a}$, the Greek indices are the spacetime coordinates while Latin indices are for the Fermi coordinates. We have $e_{0}^{\mu}=U^{\mu}$ (tangent vector) due to the geodesic equation. In the vicinity of the central geodesic, a neighbouring geodesic having separation $\xi^{\mu}$ is re-written in the Fermi basis as,

$\xi^{\mu}=Z^{i} e^{\mu}{ }_{i}$

Hence, the geodesic deviation equation in Fermi coordinates $\left(t=X^{0}, X^{i}\right)$ becomes:

$\frac{d^{2} Z^{i}}{d t^{2}}=-R_{0 j 0}^{i} Z^{j}$

$t$ and $\lambda$ both being affine parameters are related via affine transformations. Equation (9) can be obtained by substituting Eq. (8) (and also using Eq. (7)) in Eq. (6). The spatial indices associated with the frame are denoted by $i, j$ $\left(R^{i}{ }_{0 j 0}=R^{\mu}{ }_{\nu \rho \sigma} e^{i}{ }_{\mu} e^{\nu}{ }_{0} e^{\rho}{ }_{j} e^{\sigma}{ }_{0}\right)$. The tetrads and metric are related $\mathrm{via}^{1} \eta_{i j}=e^{\alpha}{ }_{i} e^{\beta}{ }_{j} g_{\alpha \beta}$.

Since the deviation has contributions both from background and gravitational radiation, we assume that the total deviation vector is decomposed in the form: $Z^{i}=Z_{B}^{i}+Z_{W}^{i}$, where the suffixes $B, W$ are for background and wave respectively. As already mentioned, a similar analysis has been carried out in [19] to separate the radiation from background curvature. The splitting of the Riemann tensor is done by noting the terms which are proportional to $H(u, x, y)$ or its derivatives $\left(R_{0 j 0}^{i}=\left(R_{0 j 0}^{i}\right)_{B}+\left(R_{0 j 0}^{i}\right)_{W}\right)$. Such terms denote the gravitational wave contribution while the other terms are due to background curvature. Thus, Eq. (9) separates into the two equations shown below.

$$
\begin{aligned}
& \frac{d^{2} Z_{B}^{i}}{d t^{2}}=-\left(R_{0 j 0}^{i}\right)_{B} Z_{B}^{j} \\
& \frac{d^{2} Z_{W}^{i}}{d t^{2}}=-\left[\left(R^{i}{ }_{0 j 0}\right)_{B}+\left(R^{i}{ }_{0 j 0}\right)_{W}\right] Z_{W}^{j}-\left(R^{i}{ }_{0 j 0}\right)_{W} Z_{B}^{j}
\end{aligned}
$$

Equation (10) is the deviation due to the background. This would have been the complete geodesic deviation equation if $H(u, x, y)=0$ in the metric line element as given in Eq. (4). Solving Eq. (11) gives the memory effect in the tetrad frame. Once both background and wave deviation (i.e. $Z_{B}^{i}$ and $Z_{W}^{i}$ ) are known, we revert back to the coordinate basis using Eq. (8) to obtain $\xi_{B}^{\mu}, \xi_{W}^{\mu}$ and $\xi^{\mu}\left(=\xi_{B}^{\mu}+\xi_{W}^{\mu}\right)$.

\footnotetext{
${ }^{1} \eta_{i j}$ denotes the Minkowski metric with signature $(-1,1,1,1)$.
} 
In the case of gyratons, we split the deviation vector as: $Z^{i}=Z_{B}^{i}+Z_{G}^{i}+Z_{W}^{i}$. Apart from the familiar terms $Z_{B}^{i}$ and $Z_{W}^{i}$, we also have deviation due to gyratons denoted by $Z_{G}^{i}$. The Riemann tensor corresponding to this deviation comprises of terms linked to $W_{i}(u, x, y)$ given in Eq. (5). The deviation equations corresponding to the three distinct effects are given by the Eqs. (64), (65) and (66) in Sect. 4.2. We then go on to calculate $\xi_{B}^{\mu}, \xi_{G}^{\mu}$ and $\xi_{W}^{\mu}$ in coordinate basis. The terms $\xi_{G}^{\mu}$ and $\xi_{W}^{\mu}$ correspond to coordinate memory effect for the gyraton and the wave respectively.

We have already emphasized the advantage of using Fermi-normal coordinates for our analysis. An important feature is that Eqs. (9), (10) and (11) take their respective forms only when the constructed tetrads are parallely transported.

It is also important to note that the results on memory from geodesic deviation are expected to match only qualitatively with those obtained from a geodesic analysis. This is because the deviation equation is perturbative by construction. In contrast there is no such restriction when we consider pairs of geodesics and differences in their separation caused by a pulse.

\section{Kundt wave metric}

We now focus on finding solutions in BD theory which represent Kundt wave spacetimes generically given as in Eq. (4). The BD scalar field is assumed to be independent of $v$ and hence, $\phi \equiv \phi(u, x, y)$. The components of Eq. (2) which are relevant for solving the field equations are listed below.

$$
\begin{aligned}
& G_{x x}=\frac{\omega}{2 \phi^{2}}\left(\phi,{ }_{x}^{2}-\phi,{ }_{y}^{2}\right) \\
& +\frac{1}{\phi}\left(\phi, x x+\frac{P, x}{P} \phi,,_{x}-\frac{P, y}{P} \phi, y\right) \\
& G_{y y}=\frac{\omega}{2 \phi^{2}}\left(\phi,,_{y}^{2}-\phi,{ }_{x}^{2}\right) \\
& +\frac{1}{\phi}\left(\phi, y y+\frac{P, y}{P} \phi, y-\frac{P, x}{P} \phi,{ }_{x}\right) \\
& G_{u u}=\frac{\omega}{\phi^{2}}\left(\phi,{ }_{u}^{2}+\frac{H}{2} P^{2}\left(\phi,{ }_{x}^{2}+\phi,{ }_{y}^{2}\right)\right) \\
& +\frac{1}{\phi}\left(\phi, u u-\frac{1}{2} P^{2} H,{ }_{x} \phi,,_{x}-\frac{1}{2} P^{2} H,{ }_{y} \phi, y\right) \\
& G_{u v}=\frac{\omega}{2 \phi^{2}} P^{2}\left(\phi,{ }_{x}^{2}+\phi,{ }_{y}^{2}\right) \\
& G_{x u}=\frac{\omega}{\phi^{2}}\left(\phi,{ }_{x} \phi,{ }_{u}\right)+\frac{1}{\phi}\left(\phi,{ }_{x u}+\frac{P, u}{P} \phi,{ }_{x}\right) \\
& G_{y u}=\frac{\omega}{\phi^{2}}(\phi, y \phi, u)+\frac{1}{\phi}\left(\phi, y u+\frac{P, u}{P} \phi, y\right)
\end{aligned}
$$

We decompose the scalar field and the metric functions as:

$$
\phi(u, x, y)=\alpha(u) \psi(x, y),
$$

$$
\begin{aligned}
P(u, x, y) & =\frac{\tilde{P}(x, y)}{U(u)}, \\
H(u, x, y) & =H^{\prime}(u) h(x, y) .
\end{aligned}
$$

Adding Eqs. (13) and (14) and using separation of variables from Eq. (18) results in $\psi_{x x}+\psi, y y=0$ (we know that $G_{x x}=G_{y y}=0$ from the metric). The solution is,

$\psi(x, y)=a+\log \left(x^{2}+y^{2}\right)$

From the metric, $G_{u v}=P^{2} \Delta \log P$ (where, $\Delta=\left(\partial_{x x}+\right.$ $\left.\left.\partial_{y y}\right)\right)$. Using this in Eq. (16) gives,

$\tilde{P}=\frac{\sqrt{x^{2}+y^{2}}}{\left[a+\log \left(x^{2}+y^{2}\right)\right]^{\omega / 2}}$

Equation (20) shows that background curvature is explicitly dependent on $\omega$. The equations for the ' $x u$ ' and ' $y u$ ' components, as in (16), (17) and given the metric (4) we end up with

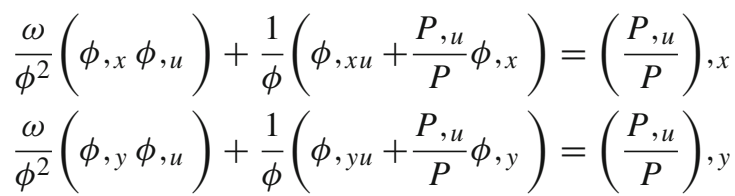

Both the above equations reduce to the same equation after using the separation of variables. We have,

$(\omega+1) \frac{\alpha, u}{\alpha}=\frac{U, u}{U}$

The Ricci scalar curvature is

$R=2 P^{2} \Delta \log P=\frac{4 \omega}{U^{2}\left[a+\log \left(x^{2}+y^{2}\right)\right]^{\omega+2}}$

The component of $G_{u u}$ from the metric is given below.

$$
\begin{aligned}
G_{u u}= & \frac{P^{2}}{2}\left(H,,_{x x}+H, y y\right)+2 \frac{P, u u}{P} \\
& -4\left(\frac{P, u}{P}\right)^{2}+H\left(-P,,_{x}^{2}-P,,_{y}^{2}+P(P, x x+P, y y)\right)
\end{aligned}
$$

Using Eqs. (15) and (18) we get

$$
\begin{aligned}
& \frac{\tilde{P}^{2}}{2 U^{2}} H^{\prime}(u)\left(h,_{x x}+h,_{y y}\right) \\
& \quad-2 \frac{U, u u}{U}+H^{\prime}(u) h\left(\frac{\tilde{P}\left(\tilde{P},_{x x}+\tilde{P},,_{y y}\right)-\tilde{P},_{x}^{2}-\tilde{P}_{, y}^{2}}{U^{2}}\right) \\
& =\omega\left[\left(\frac{\alpha, u}{\alpha}\right)^{2}+\frac{H^{\prime} h \tilde{P}^{2}}{2 U^{2}}\left(\left(\frac{\psi, x}{\psi}\right)^{2}+\left(\frac{\psi, y}{\psi}\right)^{2}\right)\right] \\
& \quad+\frac{\alpha, u u}{\alpha}-\frac{\tilde{P}^{2}}{2 U^{2}} H^{\prime}(u)\left(h,,_{x} \frac{\psi, x}{\psi}+h, \frac{\psi, y}{\psi}\right)
\end{aligned}
$$

We set $U=1$. Hence, from Eq. (23) we get $\alpha(u)$ as a constant. From Eqs. (19), (23) and (25) we find that $H^{\prime}(u)$ is 
unconstrained. The $x y$ dependent part of $H(u, x, y)$ becomes

$h(x, y)=\log \left[a+\log \left(x^{2}+y^{2}\right)\right]$

This polarization term $h(x, y)$ is different from GR. We will point out the consequences of this difference on the nature of the memory effect, contrasting it with GR. Thus from our generic analysis we find that only $H^{\prime}(u)$ and $\omega$ is unconstrained.

We now perform a coordinate transformation $x=$ $e^{X-a / 2} \cos Y, y=e^{X-a / 2} \sin Y$. The metric in the new coordinates $(u, v, X, Y)$ becomes

$d s^{2}=-H^{\prime}(u) \log (2 X) d u^{2}-2 d u d v+(2 X)^{\omega}\left(d X^{2}+d Y^{2}\right)$

The reverse transformation shows that $X=\frac{1}{2}\left(a+\log \left[\left(x^{2}+\right.\right.\right.$ $\left.\left.\left.y^{2}\right)\right]\right) \equiv \frac{1}{2} \psi[X]$. This relation shows the imprint of the scalar field in the solution of the metric, via coordinate $X$. Thus, $\phi[X(u)]$ evaluated along the geodesic $X(u)$ gives a measure of the memory effect for the scalar field due to the gravitational wave pulse.

\subsection{Displacement memory using geodesics}

The geodesic equations of coordinates $X, Y$ for the metric line element in Eq. (27) are given below.

$$
\begin{aligned}
& \frac{d^{2} X}{d u^{2}}+\frac{\omega}{2 X}\left[\left(\frac{d X}{d u}\right)^{2}-\left(\frac{d Y}{d u}\right)^{2}\right]+\frac{H^{\prime}(u)}{(2 X)^{\omega+1}}=0 \\
& \frac{d^{2} Y}{d u^{2}}+\frac{\omega}{X}\left(\frac{d X}{d u}\right)\left(\frac{d Y}{d u}\right)=0
\end{aligned}
$$

Geodesic equations for coordinate $v$ is trivial $(\ddot{u}=0)$. Hence, $u$ acts as an affine parameter. We try to solve Eqs. (28) and (29) by setting the initial value of the transverse coordinate velocities to zero (i.e. $\dot{X}=\dot{Y}=0$ ). Taking $\dot{Y}=0$ in Eq. (29) gives $\ddot{Y}=0$. Hence, $\dot{Y}=0$ for the entire evolution of the geodesic. The only non-trivial equation left is for the coordinate $X$ which is given as,

$\frac{d^{2} X}{d u^{2}}+\frac{\omega}{2 X}\left(\frac{d X}{d u}\right)^{2}+\frac{H^{\prime}(u)}{(2 X)^{\omega+1}}=0$

We use the transformation $X=\frac{1}{2} q^{\frac{1}{\omega+2}}$ for $\omega \neq 2$. Equation (30) is thus transformed to the equation

$\frac{d^{2} q}{d u^{2}}-\frac{1}{2 q}\left(\frac{d q}{d u}\right)^{2}+2(\omega+2) H^{\prime}(u)=0$

The above equation resembles that of a forced LevinsonSmith system which, generically, has an equation of the form $[54,55]$,

$\ddot{x}+a(x, \dot{x}) \dot{x}+g(x)=f(t)$ where $a(x, \dot{x}), g(x)$ and $f(t)$ need to be specified. A comparison with the equation for $q(u)$ given just above, shows the correspondence. Explicit solutions are not quite available, especially for the equation in our case, though a dynamical systems analysis exists [55].

The nature of the forcing term $H^{\prime}(u)$ (which encodes the effect of the gravitational wave pulse) dictates the behaviour of any solution. Therefore, it is likely that the analysis of such forced equations with nonlinearities may play a role in understanding the memory effect. Related discussion on the relevance of a forced equation appeared much earlier (in the context of the deviation equation) in the original work on memory by Braginsky-Grishchuk [5]. Thus, this brief side remark on the similarity of the geodesic equation for $X$ (for $\omega \neq-2$ ) with a known, nonlinear forced system, as noted above, seems worth investigating further, in future.

In principle, the geodesic equation given in Eq. (30) can be solved numerically for any value of $\omega$ for a given choice of the pulse $H^{\prime}(u)$. We have chosen $H^{\prime}(u)=\frac{1}{2} \operatorname{sech}^{2} u$ since it qualitatively resembles a gravitational wave pulse. However, as mentioned earlier, we are interested in observing memory effects for different choices of $\omega$ corresponding to different scalar curvature scenarios. The value $\omega=-2$ is special since it is the only case where the Ricci scalar is constant and negative. For other $\omega$ one may have variable positive or negative $R$. We have chosen to illustrate our analysis for the $\omega \neq-2$ case with a $\omega$ value which yields a positive but variable Ricci scalar. Results for variable negative Ricci scalar are not very different and can be worked out easily too. We discuss each case briefly with corresponding plots.

- $\underline{\omega=-2}$

We consider a scenario where the scalar curvature is constant. Hence, $\omega=-2$ and the scalar curvature is $R=-8$. The geodesic equation (30) becomes

$$
\frac{\ddot{X}}{X}-\left(\frac{\dot{X}}{X}\right)^{2}+\operatorname{sech}^{2}(u)=0
$$

One can solve Eq. (33) analytically. Setting $\dot{X} / X=p$, one finds that

$p(u)=-(1+\tanh (u))$

The constant is fixed by setting $p=0$ at $u \rightarrow-\infty$ as initially $\dot{X}$ also vanishes. Solving $X$ from the analytical form of $p(u)$ yields

$$
X(u)=\frac{A}{1+e^{2 u}}
$$


Fig. 1 Displacement memory effect for Kundt waves with $\omega=-2$

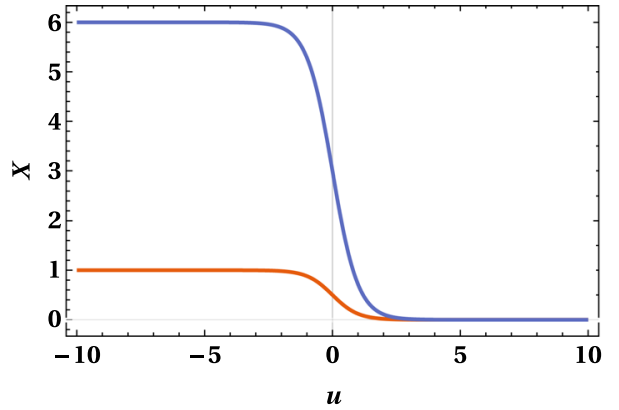

(a) Geodesics with initial

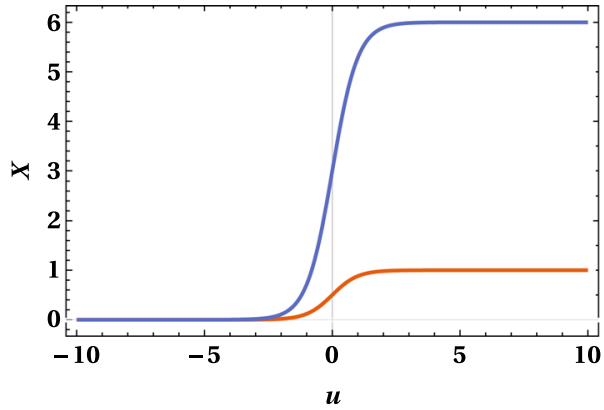

(b) Geodesics with final position as $A_{1}=1, A_{2}=6$.
Here, $A$ denotes the initial position of the particle. One can check that as $u \rightarrow-\infty, X=A$ whereas as $u \rightarrow+\infty, X=0$. Thus, two geodesics starting with different initial coordinate values, eventually have a zero $X$ value, after the passage of the pulse.

Let us now consider two different geodesics having initial $\mathrm{X}$ coordinate value as $A_{1}$ and $A_{2}$. We find, as $u \rightarrow-\infty$, $X_{1}=A_{1}, X_{2}=A_{2}$. The change in initial separation is $X_{1}-X_{2}=A_{1}-A_{2}$. The final separation at $u \rightarrow+\infty$ is zero . Thus. we have displacement memory for the coordinate $X$.

A careful inspection reveals that Eq. (33) is invariant under $u \rightarrow-u$. Hence, the analytical solution, $X(u)=$ $\frac{A}{1+e^{-2 u}}$ is also possible. Here, two geodesics both starting from $X=0$ settle to two different final values (depending on $A_{1}-A_{2}$ ). We illustrate both the analytical results below using plots.

We observe permanent displacement along X-direction (see Fig. 1). This is qualitatively similar to GR (see [46]) where we also observed constant separation after the passage of the gravitational wave pulse. Thus, for negative scalar curvature solutions, there is a qualitative agreement in the nature of the memory effect as found in GR and in $\operatorname{BD}(\omega=-2)$ theory.

- $\omega \neq-2$

The earlier geodesic analysis reveals that constant negative curvature case of $\omega=-2$ is integrable. We have not been able to find analytical solutions for other values of $\omega$. Resorting to numerics, we study the behaviour of the coordinate $X$ for $\omega=+1$. The motivation for choosing $\omega=+1$ is to compare between the results on memory for positive curvature solutions as obtained here in $\mathrm{BD}$ theory with those in GR. The Ricci scalar for $\omega=+1$ becomes $4 /\left(8 X^{3}\right)$. Thus, there is a possibility of having negative scalar curvature in $\omega=+1$ by choosing coordinate ranges where $X<0$. However, in our entire analysis of memory effects, we have restricted our coordinate range beyond the singular region so that the solution con-

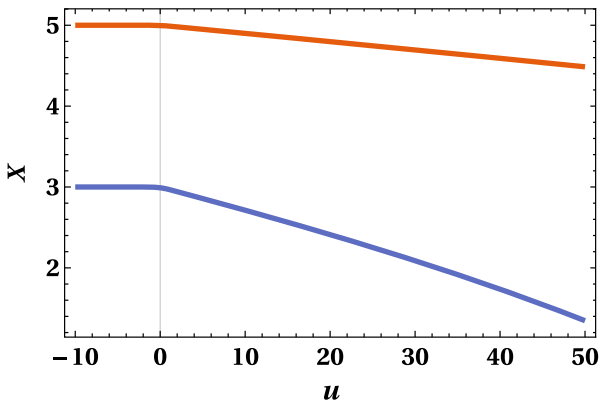

Fig. $2 \omega=1$ : Initial position of $X$ for the two geodesics are 5 (orange) and 3 (blue) respectively

forms to the positive sign of scalar curvature. We have thus avoided the negative scalar curvature region. Nevertheless, we have observed that the singularity does seem to influence pairs of geodesics through their gradual convergence towards it.

Equation (30) is solved numerically in Mathematica 10 and the evolution of coordinate $X$ is shown below.

In Fig. 2, we find increasing separation between the geodesics after the departure of the pulse. This is in sharp contrast to the profiles obtained in GR. In the latter theory we found from geodesic analysis that positive curvature scenarios give rise to a frequency memory effect [46]. This is related to the different metric functions in the Kundt wave line element for the two theories. In BD theory, $h(X, Y)=\log (2 X)$ (obtained by solving the field equations) whereas in GR we took it as $h(X, Y)=\frac{1}{2}\left(X^{2}-Y^{2}\right)$ (usual expression found in + polarization). We also find that the geodesics do not cross beyond $X=0$. This is due to the singular nature of the metric as mentioned just above.

\subsection{Geodesic deviation analysis of memory}

We now turn towards discussing geodesic deviation following the method outlined in Sect. 2.3. First, we construct an 
orthonormal tetrad for the metric line element (27).

$$
\begin{aligned}
& e_{0}{ }^{\mu}=[1, \dot{v}, \dot{X}, \dot{Y}] \\
& e_{1}^{\mu}=\left[0,-(2 X)^{\omega / 2} \dot{X},-(2 X)^{-\omega / 2}, 0\right] \\
& e_{2}{ }^{\mu}=\left[0,-(2 X)^{\omega / 2} \dot{Y}, 0,-(2 X)^{-\omega / 2}\right] \\
& e_{3}{ }^{\mu}=[-1,1-\dot{v},-\dot{X},-\dot{Y}]
\end{aligned}
$$

A similar construction was carried out in [56] for a different coordinate system. $e_{0}^{\mu}$ gives the tangent to the geodesic. The parallel transport condition (7) is only satisfied by $e_{0} \mu$ (obeys the geodesic equations) and $e_{3}{ }^{\mu}$. Both $e_{1}{ }^{\mu}, e_{2}{ }^{\mu}$ are not parallely transported. Hence, these two tetrads are rotated by an angle $\dot{\theta}_{p}=\omega \dot{Y} /(2 X)$. Since we have $\dot{Y}=0$ for all $\omega$ from the geodesic analysis, $\theta_{p}$ is a constant. We take $\theta_{p}=0$ so that the two tetrads $e_{1}^{\mu}, e_{2}{ }^{\mu}$ also satisfy Eq. (7).

The non-zero Riemann tensor components in the tetrad basis are shown below.

\section{Background}

$$
\begin{array}{lr}
\left(R_{010}^{1}\right)_{B}=\frac{\omega \dot{Y}^{2}}{2 X^{2}} \quad\left(R_{020}^{1}\right)_{B}=-\frac{\omega \dot{Y} \dot{X}}{2 X^{2}} \\
\left(R_{010}^{2}\right)_{B}=-\frac{\omega \dot{Y} \dot{X}}{2 X^{2}} \quad\left(R_{020}^{2}\right)_{B}=\frac{\omega \dot{X}^{2}}{2 X^{2}}
\end{array}
$$

Wave

$\left(R^{1} 010\right)_{W}=-\frac{(\omega+2) H^{\prime}(u)}{(2 X)^{\omega+2}}$

$\left(R^{2}{ }_{020}\right)_{W}=\frac{\omega H^{\prime}(u)}{(2 X)^{\omega+2}}$

Substituting the expressions in Eq. (37) and Eq. (38) (for the Riemann tensor components in the tetrad basis) in Eqs. (10) and (11), we solve for the background and gravitational wave contributions to the geodesic deviation.

As pointed out earlier, we ultimately go over to the coordinate basis using Eq. (8). We find out $\xi_{B}^{\mu}, \xi_{W}^{\mu}$ only along $X, Y$ directions. The total deviation $\xi^{\mu}$ is then obtained and compared with the results obtained from geodesics.

This decomposition of deviation vectors into background and wave parts can be done because the geodesic deviation equation is linear. As the geodesic equations itself are nonlinear, this method of decomposition is not possible. One may also directly integrate the geodesic deviation equation in the coordinate basis and come to similar conclusions as ours. However, using the tetrads, the equations simplify enough, as can be seen by comparing Eqs. (6) and (9). Interestingly, for $\omega=-2$ we have an exact solution.

We now show that by solving the geodesic deviation equation we may obtain the memory effect. The change in the part of the deviation vector related to the background arises due to the $P(u, x, y)$ term in Eq. (4). The change caused by the pulse $H(u, x, y)$ is conventionally related to memory, largely because the pulse is viewed as the 'cause'. Our choice of Fermi coordinates simplifies the calculations to some extent, though it is surely possible to do everything in the coordinate basis as well.

Below, we use the previously chosen $\omega$ values (as in the geodesic analysis) to carry out the deviation analysis. Since $u$ is an affine parameter we can replace the proper time $t$ mentioned earlier, with $u$.

$\bullet \omega=-2$ :

As we have seen, the geodesic equations are analytically solvable in this case. We will use the solution $X=$ $\frac{A}{1+e^{-2 u}}, \dot{Y}=0$ for further investigation. In this scenario, two geodesics starting from zero initial value have two different final separations (see Fig. 1b). Substituting $\omega=-2$ in Eqs. (37) and (38) gives a nontrivial equation only along $Z^{2}$ direction. We have,

$$
\begin{aligned}
\ddot{B} & =[1-\tanh (u)]^{2} B \\
\ddot{W} & =2[1-\tanh (u)] W+\operatorname{sech}^{2}(u) B \\
\ddot{K} & =2[1-\tanh (u)] K
\end{aligned}
$$

where $B=Z_{B}^{2}, W=Z_{W}^{2}, K=B+W$. The analytical solutions for Eqs. (39) and (41) are

$$
\begin{aligned}
B(u)= & C_{1}\left(1+e^{-2 u}\right)^{\frac{1-\sqrt{5}}{2}} \\
& \times{ }_{2} F_{1}\left[-\frac{1+\sqrt{5}}{2}, \frac{3-\sqrt{5}}{2}, 1-\sqrt{5},-\left(1+e^{-2 u}\right)\right] \\
& +C_{2}\left(1+e^{-2 u}\right)^{\frac{1+\sqrt{5}}{2}} \\
& \times{ }_{2} F_{1}\left[\frac{\sqrt{5}-1}{2}, \frac{3+\sqrt{5}}{2}, 1+\sqrt{5},-\left(1+e^{-2 u}\right)\right] \\
K(u)= & \frac{1+e^{-2 u}}{C_{3}}
\end{aligned}
$$

The solutions for $B(u)$ has hypergeometric functions denoted by ${ }_{2} F_{1} . C_{1}, C_{2}, C_{3}$ are the constants of integration. We set $\dot{K}=0$ as $u \rightarrow-\infty$ to get rid of the other constant. $W(u)$ can be easily obtained by subtracting $B(u)$ from $K(u)$. Since we start with zero initial velocities, we find $Z_{B}^{1}$ and $Z_{W}^{1}$ are constants. Equation (43) shows that the total deviation $K(u)$ is inverse of the geodesic solution $X(u)$.

Reverting back to the coordinate basis, the deviation along coordinates $X$ and $Y$ becomes

$$
\begin{aligned}
& \xi_{B}^{X}=(-2 X) Z_{B}^{1} \quad \xi_{W}^{X}=(-2 X) Z_{W}^{1} \\
& \xi_{B}^{Y}=(-2 X) B \quad \xi_{W}^{Y}=(-2 X) W
\end{aligned}
$$

Equation (44) shows how both the background and the wave deviation are simply proportional to $X$. This also follows from the Fig. 3a. Thus, the nature of the three deviations (background, wave and total) are all similar. The background and the gravitational wave both sum up to enhance the amplitude of the total deviation. Along the $Y$ direction in Fig. 3b, 
Fig. 3 Plot of deviation vectors along the coordinates $X$ and $Y$ for $\omega=-2$

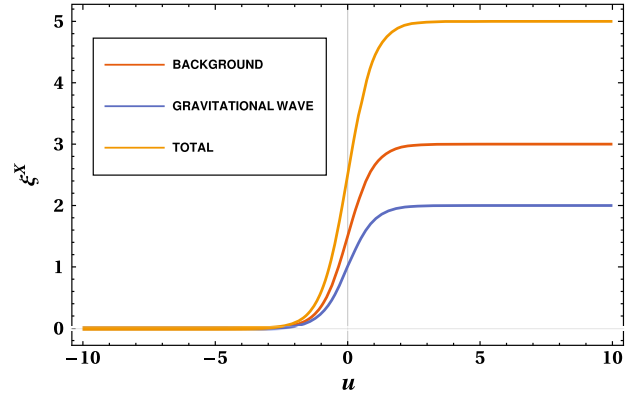

(a) $\xi^{X}$ vs $u$

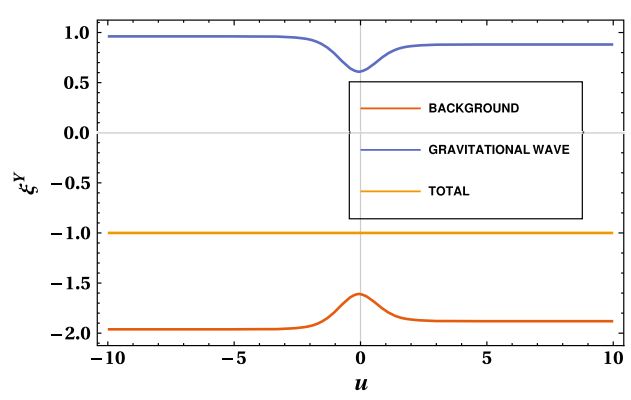

(b) $\xi^{Y}$ vs $u$ we observe that the wave and background deviations cancel each other. The total deviation is a constant. Thus, the geodesic deviation analysis also gives the same qualitative result on separation, as obtained from the geodesics. This confirms our previous assertion that the geodesic analysis can only 'see' a total deviation (or separation). It is unable to retrieve the gravitational wave contribution from the total. In both plots (Fig. 3), the blue line shows the gravitational wave memory effect.

$\bullet \omega \neq-2$ :

Similar to the geodesic analysis, we perform the deviation analysis for $\omega=+1$, which corresponds to a spacetime with a positive (but varying) Ricci scalar. The deviation equation is solved numerically. We follow the same steps as we did for $\omega=-2$. From Eq. (37), we get that $Z_{B}^{1}$ is a constant. The evolution equations of $Z_{B}^{2}, Z_{W}^{1}$ and $Z_{W}^{2}$ are solved numerically in Mathematica 10. Finally, we go over to the coordinate basis to state our results on memory.

The total deviation plot in Fig. 4b shows it to be constant, a feature also obtained from the geodesics. In Fig. 4a for the deviation vector component along the $X$ direction, we find that the deviation for the gravitational wave part is more than that for the background. The net deviation grows monotonically with the onset of the pulse. This result is consistent with the geodesic separation behaviour shown in Fig. 2. The two analyses are not precisely equivalent due to the perturbative nature of geodesic deviation. Also, we note that displacement memory is seen along both directions while frequency memory is not found.

\section{Kundt metric with gyraton terms}

Having discussed the nature of memory effect for the Kundt wave geometry in Brans-Dicke theory, in this section we will incorporate the contribution from the gyratonic terms in the generalized Kundt metric. As, discussed in Sect. 2 B, such a metric is generically given as:

$$
\begin{aligned}
d s^{2}= & -H d u^{2}-2 d u d v-2 W_{1} d u d x-2 W_{2} d u d y \\
& +\frac{1}{P^{2}}\left(d x^{2}+d y^{2}\right)
\end{aligned}
$$

where, $P \equiv P(u, x, y), H \equiv H(u, x, y)$ and the gyratonic terms $W_{i} \equiv W_{i}(u, x, y), \forall i \in\{x, y\}$.

Gyratonic spacetimes are ascribed to gravitational fields of spinning light beams $[26,27]$. The terms $W_{i}$ are responsible for angular momentum in the spacetime. Gauge transformations can locally set $W_{i}$ to zero [57]. But, globally it is not possible to remove $W_{i}$ and, thus, the spacetime retains its rotational nature.

As before, we first solve the field equations to obtain the metric functions and the scalar field and thereafter, using them, we study the memory effect via the geodesic equations and also, geodesic deviation.

From the $G^{u}{ }_{v}$ component of Eq. (2) we have $\omega \phi_{, v}^{2}+$ $\phi \phi_{, v v}=0$. We have considered a possible solution with $\phi,{ }_{v}=0$, i.e. the scalar field is independent of the null coordinate $v$. Adding the $G^{y}{ }_{x}$ and the $G^{y}{ }_{y}$ components of the Einstein tensor, we get:

$\phi_{, x x}+\phi_{, y y}=0$

which is the same as Eq. (3) in presence of a traceless matter field. In our case here, since we are considering a vacuum solution, this equation is consistent.

From the $G^{u}{ }_{u}$ component we get the following equation:

$-P^{2} \Delta \log P=-\frac{P^{2} \omega}{2 \phi^{2}}\left(\phi_{, x}^{2}+\phi_{, y}^{2}\right)$

Similar to the previous analysis, in order to solve the above equations we decompose the metric functions and the scalar field as follows:

$$
\begin{aligned}
\phi(u, x, y) & =\alpha(u) \psi(x, y), \\
P(u, x, y) & =\frac{P^{\prime}(x, y)}{U(u)}, \\
H(u, x, y) & =H^{\prime}(u) h(x, y) .
\end{aligned}
$$

Using this decomposition in Eq. (46) we get:

$$
\psi(x, y)=a+\log \left[x^{2}+y^{2}\right]
$$

Substituting the new functional forms in Eq. (47) we obtain:

$$
P^{\prime}(x, y)=\frac{\sqrt{x^{2}+y^{2}}}{\left(a+\log \left[x^{2}+y^{2}\right]\right)^{\frac{\omega}{2}}}
$$


Fig. 4 Plot of deviation vectors along the coordinates $X$ and $Y$ for $\omega=+1$

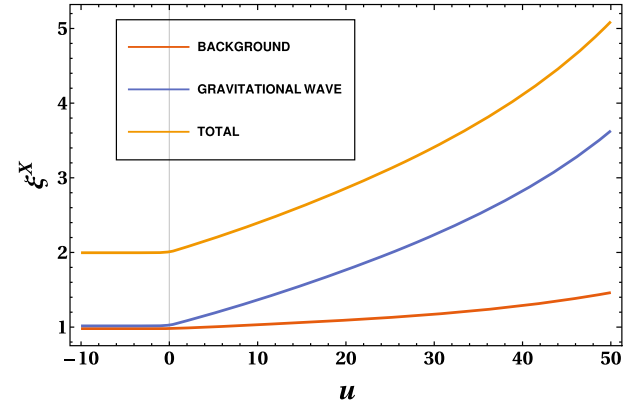

(a) $\xi^{X}$ vs $u$

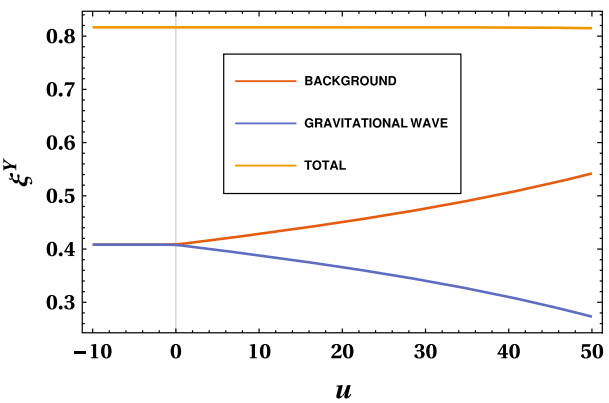

(b) $\xi^{Y}$ vs $u$
The equation that follows from the $G^{x}{ }_{u}$ component is:

$$
\begin{aligned}
{\left[\left(\frac{P_{, u}}{P}\right)_{, x}+P P_{, y}\left(W_{1, y}-W_{2, x}\right)+\frac{P^{2}}{2}\left(W_{1, y y}-W_{2, x y}\right)\right] } \\
=\left[\left(\phi_{, x} \phi_{, u}\right) \frac{\omega}{\phi^{2}}+\frac{\phi_{, x x}}{\phi}+\frac{P_{, u}}{P} \frac{\phi_{, x}}{\phi}\right. \\
\left.\quad-\frac{P^{2}}{2} \frac{\phi_{, y}}{\phi}\left(W_{1, y}-W_{2, x}\right)\right]
\end{aligned}
$$

To solve the above equation we decompose the cross terms as follows:

$W_{1}(u, x, y)=-\frac{J(u) y}{2\left[x^{2}+y^{2}\right]}$

$W_{2}(u, x, y)=\frac{J(u) x}{2\left[x^{2}+y^{2}\right]}$

where $J(u)$ is some function of $u$. We will see, while solving the geodesic equations that $J(u)$ may be associated with angular momentum.

From Eq. (51) we note that

$W_{1, y}-W_{2, x}=0$

Using the above result in Eq. (50) we find:

$\frac{\alpha_{, u}}{\alpha} \frac{\psi_{, x}}{\psi}(\omega+1)-\frac{U_{, u}}{U} \frac{\psi_{, x}}{\psi}=0$

Thus, $\alpha(u)$ and $U(u)$ satisfy:

$\frac{\alpha_{, u}}{\alpha}(\omega+1)=\frac{U_{, u}}{U}$

This is the same as the Eq. (23) in the Kundt wave metric case. The last equation that will constrain the metric function is from the $G_{u u}$ component. It leads to,

$$
\begin{gathered}
\frac{1}{8 P^{2}}\left[4 P^{4}\left(H_{, x x}+H_{, y y}\right)+8 P^{3} H\left(P_{, x x}+P_{, y y}\right)\right. \\
\left.-8 P^{2} H\left(P_{, x}^{2}+P_{, y}^{2}\right)+16 P_{, u u} P-32 P_{, u}^{2}\right] \\
=\left[\phi_{, u}^{2}+\frac{H}{2} P^{2}\left(\phi_{, x}^{2}+\phi_{, y}^{2}\right)\right] \frac{\omega}{\phi^{2}} \\
+\frac{1}{\phi}\left[\phi_{, u u}-\frac{P^{2}}{2}\left(-2 W_{1, u}+H_{, x}\right) \phi_{, x}\right.
\end{gathered}
$$

$$
\left.-\frac{P^{2}}{2}\left(-2 W_{2, u}+H_{, y}\right) \phi, y\right]
$$

From both the Eqs. (48) and (51) one can see that:

$W_{1, u} \phi_{, x}+W_{2, u} \phi_{, y}=0$

As in Kundt metric case we solve for $U(u)=$ constant. This reduces Eq. (54) to:

$h_{, x x}+h_{, y y}+\left[h_{, x} \frac{\phi, x}{\phi}+h_{, y} \frac{\phi, y}{\phi}\right]=0$

The solution is the same as for the Kundt wave case.

$H(u, x, y)=H^{\prime}(u) \log \left[a+\log \left[x^{2}+y^{2}\right]\right]$

Thus, on solving the field equations we have obtained all the metric functions except $H^{\prime}(u)$ and $J(u)$. These functions are unconstrained and are the source of the gravitational wave and the gyratonic contribution respectively. We will choose these functions judiciously while solving for the geodesics and the geodesic deviation.

Following our approach for Kundt waves, we rewrite the gyratonic metric in new coordinates defined via the coordinate transformations: $x=e^{X-a / 2} \cos Y$ and $y=$ $e^{X-a / 2} \sin Y$. As earlier, we find that the scalar $\psi$ (related to the $\phi$ ) is twice of $X$.

The metric line element in coordinates $(\mathrm{u}, \mathrm{v}, \mathrm{X}, \mathrm{Y})$ is given as:

$$
\begin{aligned}
d s^{2}= & -H^{\prime}(u) \log [2 X] d u^{2}-2 d u d v-J(u) d u d Y \\
& +(2 X)^{\omega}\left(d X^{2}+d Y^{2}\right)
\end{aligned}
$$

We can see that on comparing with the Kundt wave metric, the only difference is the presence of the $J(u) d u d Y$ term in the metric. We will see that this term will have its contribution to the overall memory effect.

\subsection{Displacement memory from geodesic analysis}

The geodesic equations corresponding to $X$ and $Y$, for the metric line element given by Eq. (57) are as follows.

$$
\frac{d^{2} X}{d u^{2}}+\frac{\omega}{2 X}\left[\left(\frac{d X}{d u}\right)^{2}-\left(\frac{d Y}{d u}\right)^{2}\right]+\frac{H^{\prime}(u)}{(2 X)^{\omega+1}}=0
$$




$$
\frac{d^{2} Y}{d u^{2}}+\frac{\omega}{X} \frac{d X}{d u} \frac{d Y}{d u}-\frac{1}{2(2 X)^{\omega}} \frac{d J(u)}{d u}=0
$$

We find that $\ddot{u}=0$ from the geodesic equation of $v$. Therefore $u$ may be chosen as as an affine parameter. From Eq. (59), it is clear that the geodesic along $Y$ coordinate is dependent on $J(u)$. This is different from the Kundt wave scenario where along $Y$ there could be no evolution. Hence, Eq. (58) is dependent on both $J(u)$ and $H^{\prime}(u)$. It is evident that the presence of cross terms involving the gyratonic contribution will have a significant impact on the evolution of the geodesics.

The displacement memory effect for coordinate $X$ obtained below is equivalent to the memory due to the scalar field, as justified earlier.

Proceeding further, we note that the first integral of Eq. (59) gives,

$\dot{Y}=\frac{C+J(u)}{2(2 X)^{\omega}}$.

Here, $C$ is a constant of integration which can be set to zero by noting that $\dot{Y}$ vanishes where $J(u)=0$. Since $X$ is functionally related to $\psi[X(u)]$, we infer from Eq. (60) that the displacement memory along coordinate $Y$ is both due to scalar field and the gyraton. As in the case without the gyraton term, we can, for $\omega \neq-2$, convert the equation for $\mathrm{X}$ to that of a generalised Levinson-Smith system with the added feature that function $g(x)$ in (32), is now $g(x, t)$. We obtain,

$$
\begin{aligned}
& \frac{d^{2} q}{d u^{2}}-\frac{1}{2 q}\left(\frac{d q}{d u}\right)^{2}+-\frac{\omega(\omega+2)}{4 q^{\frac{\omega}{\omega+2}}} J^{2}(u) \\
& +2(\omega+2) H^{\prime}(u)=0
\end{aligned}
$$

Asymptotic behaviour of Eq. (61) has been studied in [58]. Such solutions are bounded and converge as $u \rightarrow+\infty$.

Let us now separately deal with the constant curvature solution $(\omega=-2)$ and singular solutions $(\omega \neq-2)$. The Ricci scalar in each case turns out to be the same as for the Kundt wave metric. We assume $H^{\prime}(u)=\frac{\operatorname{sech}^{2}(u)}{2}$ (same as in the Kundt wave metric) and $J(u)=b \operatorname{sech}^{2}(u){ }^{2}$ Thus, both the wave and gyratonic terms are dominant for a limited and finite duration of "time" (i.e. $u$ ).

- $\omega=-2$

This case corresponds to $R=-8$, a constant negative curvature solution. We solve the geodesic equations (58) and (59) for this $\omega$ numerically in Mathematica 10 and plot the solutions.

The geodesic evolution show a constant shift along $u$ as well as a displacement memory effect along both $X$ and $Y$ directions. Apart from the shift along $u$ (which is present

${ }^{2}$ We will set the parameter $b=1$ for our analysis. in the evolution of both $X$ and $Y$ ), Fig. 5b, i.e. the evolution of $Y$, also shows the role the gyratonic terms in the metric. The plot along the $X$ direction in Fig. 5a is similar (but shifted along $u$ ) to the Kundt wave case.

- $\omega \neq-2$

Since the Ricci scalar is the same as for the Kundt wave metric, we find that for $\omega \neq 2$ there is a singularity at $X=0$. We have carefully restricted our geodesic analysis in the domain $X>0$. The plots for the geodesics are shown below.

From Fig. 6 we see that the separation slowly builds up along the $X$ direction. We note from Eq. (60) that $\dot{Y}=J(u) /(4 X)$. Since $J(u)$ is a sech-squared pulse, we find that $\dot{Y}$ is zero at $u \rightarrow \pm \infty$. The only significant contribution of the gyratonic pulse is centered at $u=0$. Thus, for the gyratonic Kundt metric, both the $X$ and $Y$ coordinates have memory effect around $u=0$ in contrast to memory only along the $X$ direction for Kundt waves.

From the plots of the geodesics it is clear that the most visible contribution of the gyratonic term is in the evolution along for $Y$. As for the evolution along $\mathrm{X}$, the plots are shifted because of the additional term dependent on $\dot{Y}^{2} \propto J[u]^{2}$ in the Eq. (58).

\subsection{Geodesic deviation analysis of the memory effect}

The orthonormal tetrads for the gyratonic Kundt metric (57) are written below.

$$
\begin{aligned}
e_{0}{ }^{\mu} & =[1, \dot{v}, \dot{X}, \dot{Y}] \quad e_{3}{ }^{\mu}=[-1,1-\dot{v},-\dot{X},-\dot{Y}] \\
e_{1}{ }^{\mu} & =\left[0,-(2 X)^{\omega / 2} \dot{X},-(2 X)^{-\omega / 2}, 0\right] \\
e_{2}{ }^{\mu} & =\left[0,-(2 X)^{\omega / 2} \dot{Y}+(J / 2)(2 X)^{-\omega / 2}, 0,-(2 X)^{-\omega / 2}\right]
\end{aligned}
$$

The gyratonic term $J(u)$ is only present in $e_{2}{ }^{\mu}$. We now check whether the constructed tetrads are parallely transported, i.e. $\left(U^{\mu}=e_{0}^{\mu}\right)$.

$$
\begin{aligned}
& U \cdot D{e_{0}}^{\mu}=0, \quad U \cdot D e_{1}^{\mu}=\frac{\omega \dot{Y}}{2 X} e_{2}^{\mu}, \\
& U \cdot D e_{2}^{\mu}=-\frac{\omega \dot{Y}}{2 X} e_{1}^{\mu}, \quad U \cdot D e_{3}^{\mu}=0
\end{aligned}
$$

Therefore, if we rotate $e_{1}{ }^{\mu}$ and $e_{2}{ }^{\mu}$ by an angle $\theta_{p}$, such that it satisfies $\dot{\theta_{p}}=\frac{\omega \dot{Y}}{2 X}$, we will obtain a parallely propagating tetrad. Thus, $\theta_{p}$ is dependent on the gyratonic term $J(u)$, following from Eq. (60). In the new tetrad basis, we calculate the nonvanishing Riemann components and separate them into background, gyratonic and the gravitational wave parts. The deviation equation for the three parts are

$$
\frac{d^{2} Z_{B}^{i}}{d u^{2}}=-\left(R_{0 j 0}^{i}\right)_{B} Z_{B}^{j}
$$


Fig. 5 Geodesics for gyratonic Kundt metric with $\omega=-2$
Fig. 6 Geodesics for gyratonic Kundt metric with $\omega=+1$

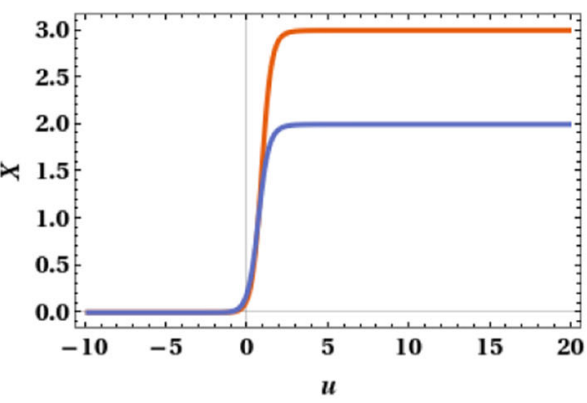

(a) Final positions of $\mathrm{X}$ are 3 (orange) and 2 (blue) respectively.

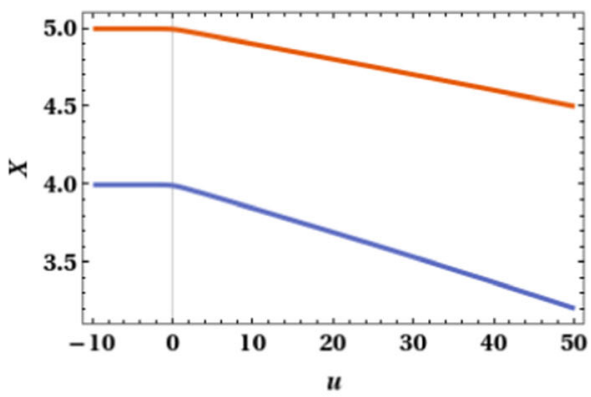

(a) Initial positions of $X$ are 5 (orange) and 4 (blue) respectively.

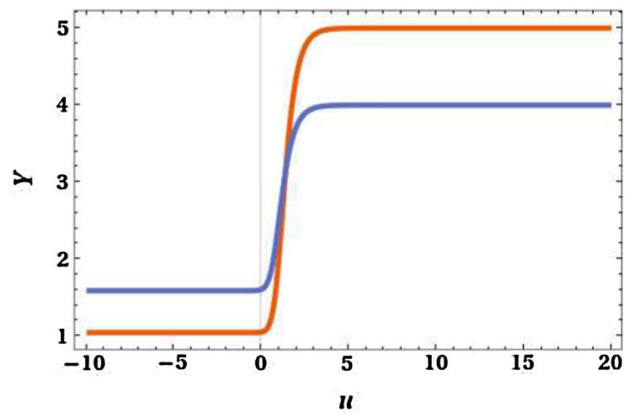

(b) Initial positions of $Y$ are 1 (orange) and 2 (blue) respectively.

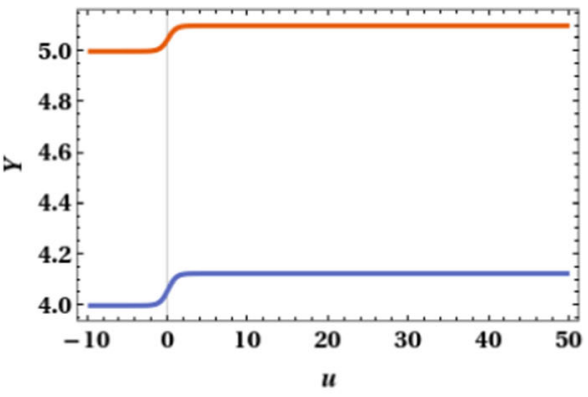

(b) Initial positions of $Y$ are 5 (orange) and 4 (blue) respectively.

$$
\begin{aligned}
\frac{d^{2} Z_{G}^{i}}{d u^{2}}= & -\left[\left(R^{i}{ }_{0 j 0}\right)_{B}+\left(R_{0 j 0}^{i}\right)_{G}\right] Z_{G}^{j}-\left(R^{i}{ }_{0 j 0}\right)_{G} Z_{B}^{j} \\
\frac{d^{2} Z_{W}^{i}}{d u^{2}}= & -\left[\left(R_{0 j 0}^{i}\right)_{B}+\left(R_{0 j 0}^{i}\right)_{G}+\left(R_{0 j 0}^{i}\right)_{W}\right] Z_{W}^{j} \\
& -\left(R^{i}{ }_{0 j 0}\right)_{W}\left(Z_{B}^{j}+Z_{G}^{j}\right) .
\end{aligned}
$$

The deviation equations for the gyratonic Kundt metric are straightforward generalizations of Eqs. (10) and (11) where there was no contribution coming from the term $\left(R^{i}{ }_{0 j 0}\right)_{G}$. The expressions for the Riemann tensor components in the tetrad frame for these three components are given in the Appendix [see Eqs. (72)-(80)]. The geodesic deviation equations (64), (65) and (66) were numerically solved in Mathematica 10 and the deviation vectors were obtained first in the tetrad basis. Specific initial values are assumed for each part of the deviation vector (i.e. background, wave and gyraton) at a $u$ value reasonably far from where the pulse $H^{\prime}(u)$ or the gyratonic term $J(u)$ is significant (i.e. near $u=0$ ). Thereafter, following the procedure adopted for the Kundt waves, the deviation vectors are transformed back to the coordinate basis where the evolution is analysed. The plots thus generated appear in Figs. 7, 8, 9 and 10 for $\omega=-2$ and $\omega=1$ respectively.

The evolution of each part of the deviation vector (i.e. background, wave and gyraton) as shown explicitly in Figs. 7 and 9, demonstrate their individual contributions. The qualitative similarity of the total deviation plots (Figs. 8, 10) with the results found using geodesics (Figs. 5, 6) is quite evident. On comparing the plots for deviation in Kundt wave metric (Figs. 3, 4) with deviation plots for the gyratonic Kundt metric we note that along the $X$ direction, the plots are very similar, except for the additional gyratonic contribution. However, along the $Y$ direction the plots are significantly different. In the Kundt wave case, background and gravitational wave contributions cancel each other, whereas in the gyratonic Kundt metric, the contribution sums up and gives an effective displacement memory.

\section{Conclusions}

Let us now summarise pointwise, the main results obtained in this article.

- In vacuum Brans-Dicke theory we have constructed solutions representing a Kundt-type line element without and with gyratonic terms. The solutions have the expected feature of two unspecified functions $H^{\prime}(u)$ and $J(u)$ which represent the wave-profile and the gyraton term respectively. Both these functions can be specified while writing down explicit solutions. We choose them to be 
Fig. 7 Deviation plots for $\omega=-2$

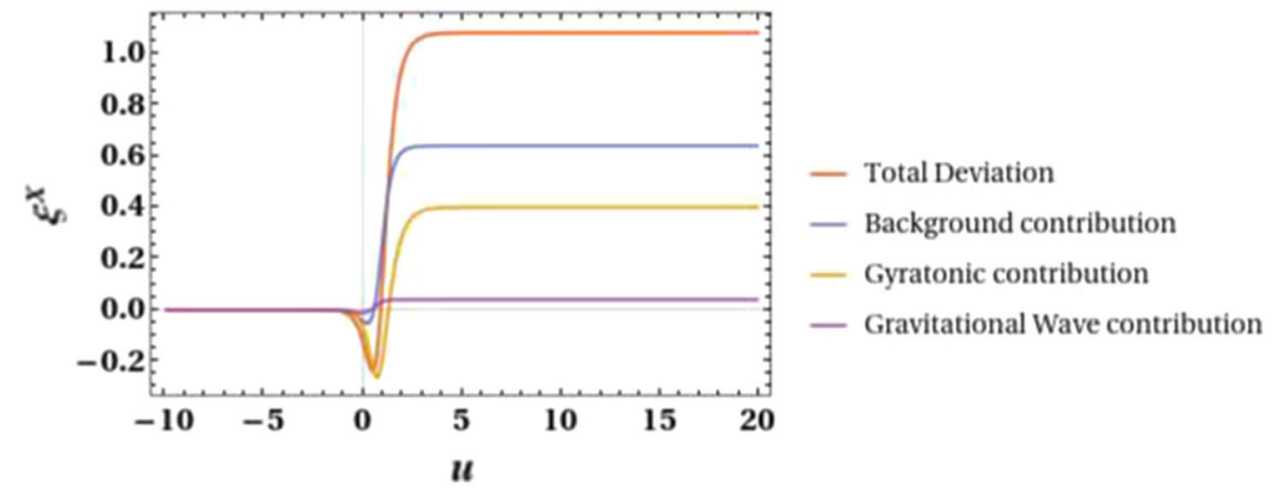

(a) Deviation along $X$.

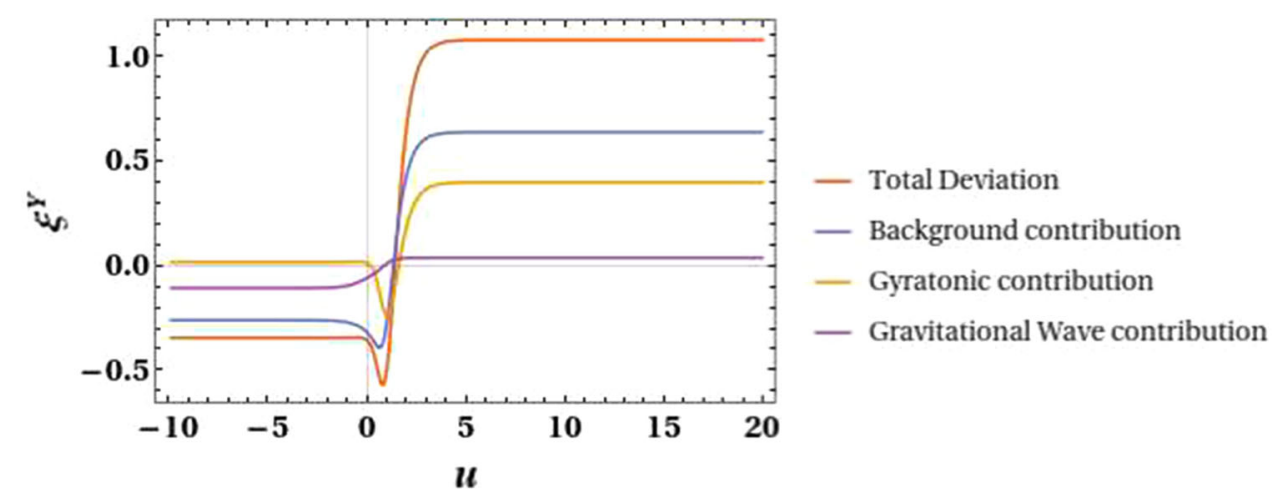

(b) Deviation along $Y$.

Fig. 8 Total deviation plots for $\omega=-2$

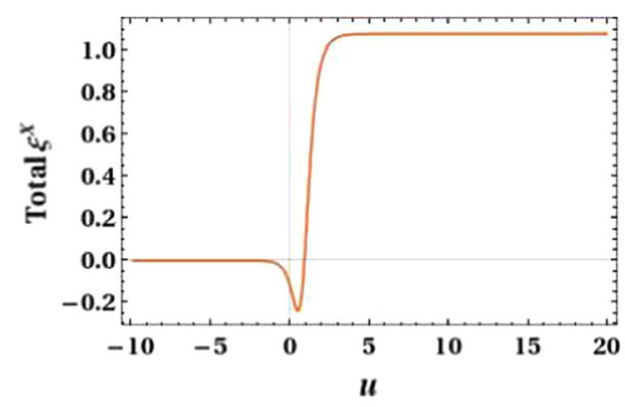

(a) Total deviation along $X$

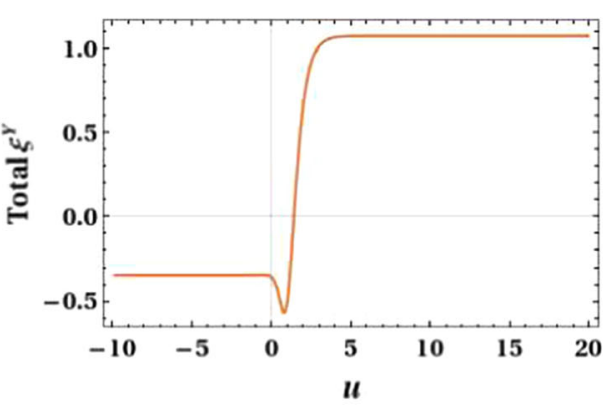

(b) Total deviation along $Y$. localised fucntions (eg. proportional to $\operatorname{sech}^{2} u$ ) with the aim of studying memory effects.

- For both line elements with chosen profiles for the $H^{\prime}(u)$ and $J(u)$ we first study geodesics and then geodesic deviation in order to obtain memory effects. Logical similarities between results using geodesics and geodesic deviation are visible in our results. Displacement memory via geodesics and memory found using solutions of the deviation equation explicitly demonstrate the dependencies on the presence and profiles of the functions $H^{\prime}$ and $J$. The role of the gyraton term is clearly visible in the memory effects.
- Displacement memory for $\omega=-2$ and without the gyraton term is exactly solvable and easy to understand analytically-a feature we show while analysing this specific case with constant negative Ricci scalar.

- In our analysis of deviation we have used a parallely transported Fermi basis where the equations simplify. We have split the deviation vector into background, gravitational wave and gyratonic parts and have shown how each part influences the behaviour of the total deviation vector, thereby ensuring the existence of memory.

- The identification of the main geodesic equation for general $\omega$ with a known dynamical system, the LevinsonSmith system, is an interesting observation in our work. 
Fig. 9 Deviation Plots for $\omega=1$

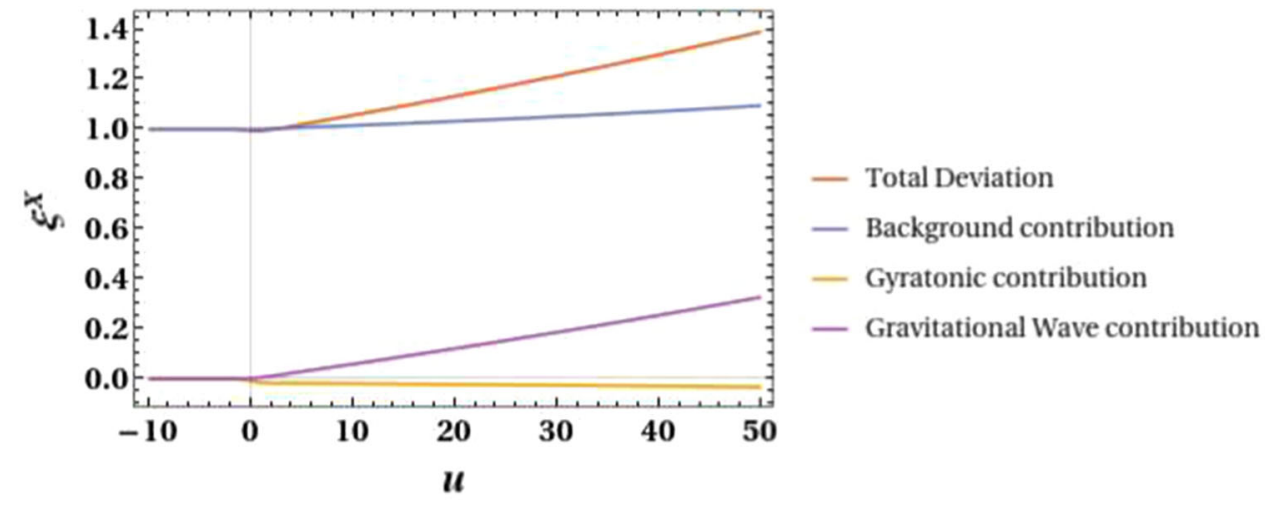

(a) Deviation along $X$.

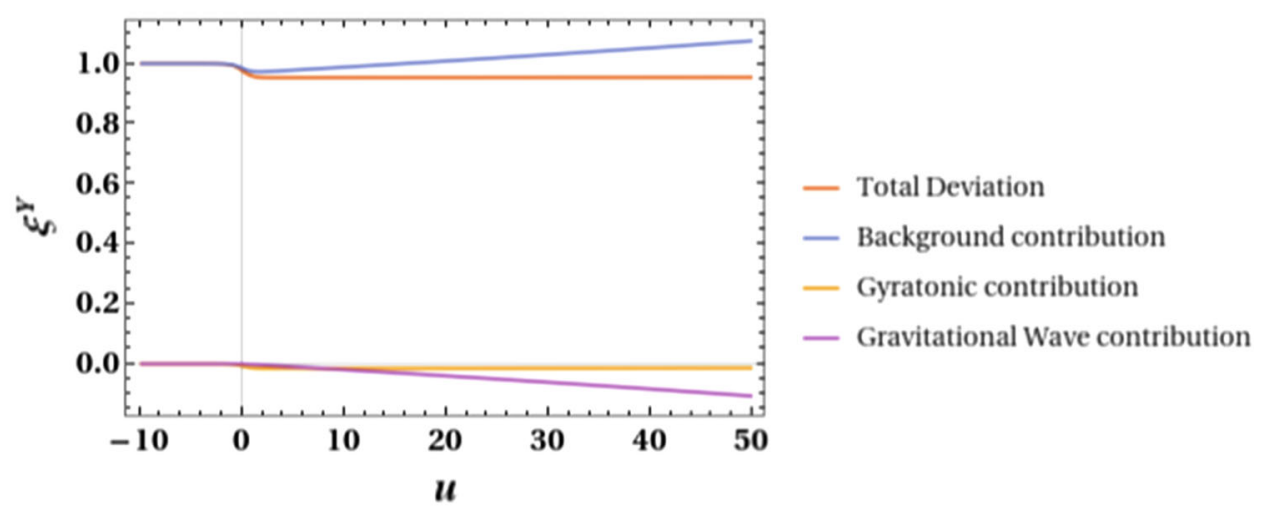

(b) Deviation along $Y$.

Fig. 10 Total deviation plots for $\omega=1$

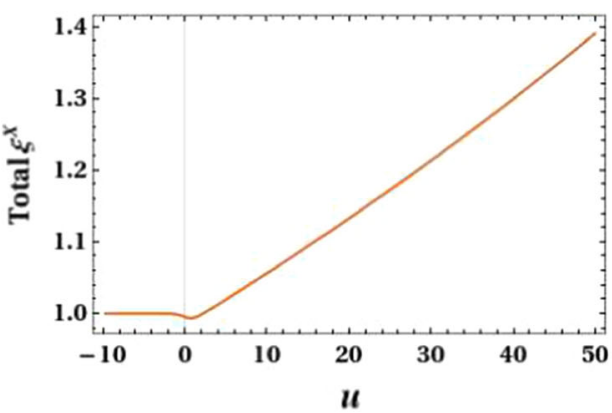

(a) Total deviation along $X$

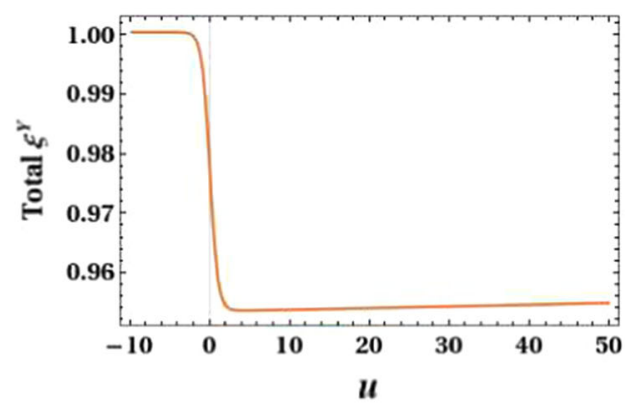

(b) Total deviation along $Y$.
The lack of exact solutions of such systems was a hindrance in using this correspondence for understanding memory. As mentioned earlier, it may be possible to extend and enrich our work along these lines using inputs from mathematics and the theory of dynamical systems.

The memory effects shown here for the two different Kundt metrics are characteristic features of BD theory itself. This is apparent through the dependencies of memory on $\omega$ and/or the BD scalar field. Thus, there is an intrinsic difference with similar scenarios in GR at a qualitative as well as a quantitative level.

The geodesic deviation analysis carried out here (using the Fermi basis etc.) can readily be applied to calculate memory effects for any spacetime, in particular those where radiative behaviour is present. Further, one may also employ the $\mathcal{B}$ matrix formalism as introduced in [59] to study the behaviour of the kinematic variables of timelike geodesic congruences. One may also search for memories in impulsive gravitational wave spacetimes $[60,61]$ and compare with the results obtained here, by setting appropriate limits. 
Finally, it may be worthwhile identifying Kundt geometries in other alternative theories so that a comparison can be made on the nature and distinguishing features of the memory effects manifest in each such theory. Even within BD theory, there exists scope of finding newer Kundt spacetimes for which memory effects can always be investigated using the methods outlined here. We hope to return to these issues in future work.

Acknowledgements SS thanks the Department of Physics, IIT Kharagpur, India for providing him with the opportunity to work on this project during his tenure as an integrated M.Sc. student. IC acknowledges Matthias Blau for discussions. IC also thanks the University Grants Commission (UGC), Government of India for providing financial assistance through a senior research fellowship (SRF) (reference ID: 523711).

Data Availability Statement This manuscript has no associated data or the data will not be deposited. [Authors' comment: This article is entirely theoretical. No data from any other source is used anywhere, in the paper. Hence, no extra data needs to be deposited.]

Open Access This article is licensed under a Creative Commons Attribution 4.0 International License, which permits use, sharing, adaptation, distribution and reproduction in any medium or format, as long as you give appropriate credit to the original author(s) and the source, provide a link to the Creative Commons licence, and indicate if changes were made. The images or other third party material in this article are included in the article's Creative Commons licence, unless indicated otherwise in a credit line to the material. If material is not included in the article's Creative Commons licence and your intended use is not permitted by statutory regulation or exceeds the permitted use, you will need to obtain permission directly from the copyright holder. To view a copy of this licence, visit http://creativecomm ons.org/licenses/by/4.0/.

Funded by SCOAP 3 .

\section{Appendix}

\section{Einstein tensor components}

The Einstein tensor components of the generalized Kundt metric with gyratons are given below.

$$
\begin{aligned}
G^{u}{ }_{v}= & G^{x}{ }_{x}=G_{y}^{y}=0 \\
G^{u}{ }_{u}= & -P^{2} \Delta \log [P] \\
G^{x}{ }_{u}= & {\left[\left(\frac{P_{, u}}{P}\right)_{, x}+P_{, y}\left(W_{1, y}-W_{2, x}\right)\right.} \\
& \left.+\frac{P^{2}}{2}\left(W_{1, y y}-W_{2, x y}\right)\right] \\
G^{y}= & {\left[\left(\frac{P_{, u}}{P}\right)_{, y}+P P_{, y}\left(W_{2, x}-W_{1, y}\right)\right.} \\
& \left.+\frac{P^{2}}{2}\left(W_{2, x x}-W_{1, x y}\right)\right]
\end{aligned}
$$

$$
\begin{aligned}
G_{u u}= & \frac{1}{8 P^{2}}\left[4 P^{4}\left(H_{, x x}+H_{, y y}\right)+8 P^{3} H\left(P_{, x x}+P_{, y y}\right)\right. \\
& \left.-8 P^{2} H\left(P_{, x}^{2}+P_{, y}^{2}\right)+16 P_{, u u} P-32 P_{, u}^{2}\right]
\end{aligned}
$$

Note that by setting $W_{1}=W_{2}=0$ we get the Einstein tensors for the Kundt wave metric given in Eq. (4).

2. Riemann tensor in tetrad frame for Kundt spacetimes with gyraton terms

The Riemann tensor in the parallely propagated tetrad frame for the gyratonic Kundt metric are as follows.

\section{Background}

$$
\begin{aligned}
\left(R^{1}{ }_{010}\right)_{B}= & \frac{\omega}{2 X^{2}}\left[\sin \left(\theta_{p}\right) \dot{X}+\cos \left(\theta_{p}\right) \dot{Y}\right]^{2} \\
\left(R^{1}{ }_{020}\right)_{B}= & \left(R^{2}{ }_{010}\right)_{B}=-\frac{\omega}{4 X^{2}}\left[\sin \left(2 \theta_{p}\right)\left(\dot{X}^{2}-\dot{Y}^{2}\right)\right. \\
& \left.+2 \cos \left(2 \theta_{p}\right) \dot{X} \dot{Y}\right] \\
\left(R^{2}{ }_{020}\right)_{B}= & \frac{\omega}{2 X^{2}}\left[\cos \left(\theta_{p}\right) \dot{X}-\sin \left(\theta_{p}\right) \dot{Y}\right]^{2}
\end{aligned}
$$

\section{Gyraton}

$$
\begin{aligned}
& \left(R_{010}^{1}\right)_{G}=-\frac{\omega X}{(2 X)^{\omega+2}} \dot{J}(u) \sin \left(2 \theta_{p}\right) \\
& \left(R^{1}{ }_{020}\right)_{G}=\left(R_{010}^{2}\right)_{G}=\frac{\omega X}{(2 X)^{\omega+2}} \dot{J}(u) \cos \left(2 \theta_{p}\right) \\
& \left(R^{2}{ }_{020}\right)_{G}=\frac{\omega X}{(2 X)^{\omega+2}} \dot{J}(u) \sin \left(2 \theta_{p}\right)
\end{aligned}
$$

\section{Gravitational wave}

$$
\begin{aligned}
& \left(R_{010}^{1}\right)_{W}=-\frac{H^{\prime}(u)}{(2 X)^{\omega+2}}\left[1+(\omega+1) \cos \left(2 \theta_{p}\right)\right] \\
& \left(R_{020}^{1}\right)_{W}=\left(R^{2}{ }_{010}\right)_{W}=-\frac{\omega+1}{(2 X)^{\omega+2}} H^{\prime}(u) \sin \left(2 \theta_{p}\right) \\
& \left(R_{020}^{2}\right)_{W}=\frac{H^{\prime}(u)}{(2 X)^{\omega+2}}\left[(\omega+1) \cos \left(2 \theta_{p}\right)-1\right]
\end{aligned}
$$

An overdot denotes differentiation w.r.t. $u$.

\section{References}

1. B. Abbott et al. (LIGO Scientific, Virgo), Phys. Rev. Lett. 116, 061102 (2016) https://doi.org/10.1103/PhysRevLett.116.061102

2. E. Berti, K. Yagi, N. Yunes, Gen. Relativ. Gravit. 50, 46 (2018). https://doi.org/10.1007/s10714-018-2362-8

3. M. Favata, Class. Quantum Gravity 27, 084036 (2010). https://doi. org/10.1088/0264-9381/27/8/084036

4. Y.B. Zel'dovich, A.G. Polnarev, Sov. Astron. 18, 17 (1974)

5. V.B. Braginsky, L.P. Grishchuk, Sov. Phys. JETP 62, 427 (1985)

6. M. Ludvigsen, Gen. Relativ. Gravit. 21, 1205 (1989). https://doi. org/10.1007/BF00763308 
7. D. Christodoulou, Phys. Rev. Lett. 67, 1486 (1991). https://doi.org/ 10.1103/PhysRevLett.67.1486

8. K.S. Thorne, Phys. Rev. D 45, 520 (1992). https://doi.org/10.1103/ PhysRevD.45.520

9. L. Bieri, D. Garfinkle, Class. Quantum Gravity 30, 195009 (2013). https://doi.org/10.1088/0264-9381/30/19/195009

10. M. Pate, A.-M. Raclariu, A. Strominger, Phys. Rev. Lett. 119, 261602 (2017). https://doi.org/10.1103/PhysRevLett.119.261602

11. N. Jokela, K. Kajantie, M. Sarkkinen, Phys. Rev. D 99, 116003 (2019). https://doi.org/10.1103/PhysRevD.99.116003

12. A. Strominger, A. Zhiboedov, J. High Energy Phys. 01, 86 (2016). https://doi.org/10.1007/JHEP01(2016)086

13. A. Strominger, Lectures on the Infrared Structure of Gravity and Gauge Theory (2017). arXiv:1703.05448 [hep-th]

14. P.-M. Zhang, C. Duval, G.W. Gibbons, P.A. Horvathy, Phys. Lett. B 772, 743 (2017). https://doi.org/10.1016/j.physletb.2017.07.050

15. I. Chakraborty, S. Kar, Phys. Rev. D 101, 064022 (2020). https:// doi.org/10.1103/PhysRevD.101.064022

16. E.E. Flanagan, A.M. Grant, A.I. Harte, D.A. Nichols, Phys. Rev. D 99, 084044 (2019). https://doi.org/10.1103/PhysRevD.99.084044

17. Y. Hamada, M.-S. Seo, G. Shiu, Phys. Rev. D 96, 023509 (2017). https://doi.org/10.1103/PhysRevD.96.023509

18. L. Bieri, D. Garfinkle, N. Yunes, Class. Quantum Gravity 34, 215002 (2017). https://doi.org/10.1088/1361-6382/aa8b52

19. C.-S. Chu, Y. Koyama, Phys. Rev. D 100, 104034 (2019). https:// doi.org/10.1103/PhysRevD.100.104034

20. W. Kundt, Z. Phys. 163, 77 (1961). https://doi.org/10.1007/ BF01328918

21. H. Stephani, D. Kramer, M.A.H. MacCallum, C. Hoenselaers, E. Herlt, Exact Solutions of Einstein's Field Equations (Cambridge University Press, Cambridge, 2003). https://doi.org/10. 1017/CBO9780511535185

22. J.B. Griffiths, J. Podolsky, Exact Space-Times in Einstein's General Relativity (Cambridge University Press, Cambridge, 2009). https:// doi.org/10.1017/CBO9780511635397

23. H.W. Brinkmann, Math. Ann. 94, 119 (1925). https://doi.org/10. 1007/BF01208647

24. N. Rosen, Phys. Z. Sowjetunion 12, 366 (1937)

25. J. Podolský, M. Ortaggio, Class. Quantum Gravity 20, 1685 (2003). https://doi.org/10.1088/0264-9381/20/9/307

26. V.P. Frolov, D.V. Fursaev, Phys. Rev. D 71, 104034 (2005). https:// doi.org/10.1103/PhysRevD.71.104034

27. V.P. Frolov, W. Israel, A. Zelnikov, Phys. Rev. D 72, 084031 (2005). https://doi.org/10.1103/PhysRevD.72.084031

28. H. Kadlecová, A. Zelnikov, P. Krtouš, J. Podolský, Phys. Rev. D 80, 024004 (2009). https://doi.org/10.1103/PhysRevD.80.024004

29. J. Podolský, M. Ortaggio, Class. Quantum Gravity 18, 2689 (2001). https://doi.org/10.1088/0264-9381/18/14/307

30. M. Ortaggio, J. Podolský, Class. Quantum Gravity 19, 5221 (2002). https://doi.org/10.1088/0264-9381/19/20/313

31. J.B. Griffiths, P. Docherty, J. Podolský, Class. Quantum Gravity 21, 207 (2003). https://doi.org/10.1088/0264-9381/21/1/014

32. A. Coley, S. Hervik, G. Papadopoulos, N. Pelavas, Class. Quantum Gravity 26, 105016 (2009). https://doi.org/10.1088/0264-9381/ 26/10/105016

33. J. Podolský, R. Švarc, Class. Quantum Gravity 30, 205016 (2013). https://doi.org/10.1088/0264-9381/30/20/205016
34. T. Tahamtan, O. Svítek, Eur. Phys. J. C 77, 384 (2017). https://doi. org/10.1140/epjc/s10052-017-4945-z

35. R. Svarc, J. Podolský, O. Hruška, Phys. Rev. D 102, 084012 (2020). https://doi.org/10.1103/PhysRevD.102.084012

36. V. Pravda, A. Pravdová, J. Podolský, R. Švarc, Phys. Rev. D 95, 084025 (2017). https://doi.org/10.1103/PhysRevD.95.084025

37. C.M. Will, Living Rev. Relativ. 17, 4 (2014). https://doi.org/10. 12942/lrr-2014-4

38. R.N. Lang, Phys. Rev. D 89, 084014 (2014). https://doi.org/10. 1103/PhysRevD.89.084014

39. R.N. Lang, Phys. Rev. D 91, 084027 (2015). https://doi.org/10. 1103/PhysRevD.91.084027

40. S.M. Du, A. Nishizawa, Phys. Rev. D 94, 104063 (2016). https:// doi.org/10.1103/PhysRevD.94.104063

41. K. Koyama, Phys. Rev. D 102, 021502 (2020). https://doi.org/10. 1103/PhysRevD.102.021502

42. S. Tahura, D.A. Nichols, A. Saffer, L.C. Stein, K. Yagi, arXiv:2007.13799 [gr-qc]

43. S. Hou, in 9th International Workshop on Astronomy and Relativistic Astrophysics (2020). https://doi.org/10.1002/asna.2021.13887

44. H. Bondi, M.G.J. van der Burg, A.W.K. Metzner, Proc. R. Soc. Lond. A 269, 21 (1962). https://doi.org/10.1098/rspa.1962.0161

45. S. Hou, Z.-H. Zhu, Chin. Phys. C 45, 023122 (2021). https://doi. org/10.1088/1674-1137/abd087

46. I. Chakraborty, S. Kar, Phys. Lett. B 808, 135611 (2020). https:// doi.org/10.1016/j.physletb.2020.135611

47. P.-M. Zhang, C. Duval, G.W. Gibbons, P.A. Horvathy, Phys. Rev. D 96, 064013 (2017). https://doi.org/10.1103/PhysRevD.96.064013

48. G.M. Shore, JHEP 2018(12), 133 (2018). https://doi.org/10.1007/ JHEP12(2018)133

49. C. Brans, R. Dicke, Phys. Rev. 124, 925 (1961). https://doi.org/10. 1103/PhysRev.124.925

50. S. Hou, Y. Gong, Eur. Phys. J. C 78, 247 (2018). https://doi.org/ 10.1140/epjc/s10052-018-5738-8

51. G.T. Horowitz (1992). arXiv:hep-th/9210119

52. V. Faraoni, Cosmology in Scalar Tensor Gravity (Kluwer Academic Publishers, Dordecht, 2004). https://doi.org/10.1007/ 978-1-4020-1989-0

53. F.K. Manasse, C.W. Misner, J. Math. Phys. 4, 735 (1963). https:// doi.org/10.1063/1.1724316

54. N. Levinson, O.K. Smith, Duke Math. J. 9, 382 (1942). https://doi. org/10.1215/S0012-7094-42-00928-1

55. A.P. Krishchenko, Ord. Differ. Equ. 55, 1431 (2019). https://doi. org/10.1134/S001226611911003X

56. J. Bicak, J. Podolsky, J. Math. Phys. 40, 4495 (1999). https://doi. org/10.1063/1.532981

57. J. Podolský, R. Steinbauer, R. Švarc, Phys. Rev. D 90, 044050 (2014). https://doi.org/10.1103/PhysRevD.90.044050

58. C. Tunç, E. Tunç, J. Frankl. Inst. 344, 391 (2007). https://doi.org/ 10.1016/j.jfranklin.2006.02.011

59. M. O'Loughlin, H. Demirchian, Phys. Rev. D 99, 024031 (2019). https://doi.org/10.1103/PhysRevD.99.024031

60. P.-M. Zhang, C. Duval, P.A. Horvathy, Class. Quantum Gravity 35, 065011 (2018). https://doi.org/10.1088/1361-6382/aaa987

61. S. Bhattacharjee, S. Kumar, A. Bhattacharyya, Phys. Rev. D 100 , 084010 (2019). https://doi.org/10.1103/PhysRevD.100.084010 